\title{
Real power loss diminution by predestination of particles wavering search algorithm
}

\author{
Kanagasabai Lenin \\ Department of EEE, Prasad V. Potluri Siddhartha Institute of Technology, India
}

\begin{tabular}{l} 
Article Info \\
\hline Article history: \\
Received Nov 15, 2019 \\
Revised Jan 17, 2020 \\
Accepted Feb 11, 2020 \\
\hline
\end{tabular}

\section{Keywords:}

Optimal reactive power Predestination of particles wavering search algorithm Transmission loss

\begin{abstract}
In this work Predestination of Particles Wavering Search (PPS) algorithm has been applied to solve optimal reactive power problem. PPS algorithm has been modeled based on the motion of the particles in the exploration space. Normally the movement of the particle is based on gradient and swarming motion. Particles are permitted to progress in steady velocity in gradientbased progress, but when the outcome is poor when compared to previous upshot, immediately particle rapidity will be upturned with semi of the magnitude and it will help to reach local optimal solution and it is expressed as wavering movement. In standard IEEE 14, 30, 57,118,300 bus systems Proposed Predestination of Particles Wavering Search (PPS) algorithm is evaluated and simulation results show the PPS reduced the power loss efficiently.
\end{abstract}

This is an open access article under the CC BY-SA license.

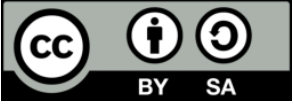

\section{Corresponding Author:}

Kanagasabai Lenin,

Department of EEE,

Prasad V. Potluri Siddhartha Institute of Technology,

Kanuru, Vijayawada, Andhra Pradesh -520007, India.

Email: gklenin@gmail.com

\section{INTRODUCTION}

Reactive power problem plays a key role in secure and economic operations of power system. Optimal reactive power problem has been solved by variety of types of methods [1-6]. Nevertheless numerous scientific difficulties are found while solving problem due to an assortment of constraints. Evolutionary techniques [7-15] are applied to solve the reactive power problem, but the main problem is many algorithms get stuck in local optimal solution \& failed to balance the Exploration \& Exploitation during the search of global solution. In this work, Predestination of Particles Wavering Search (PPS) algorithm has been applied to solve optimal reactive power problem. PPS algorithm has been modeled based on the motion of the particles in the exploration space. Particles will arbitrarily move in the exploration space in many algorithms which has been already applied to many optimization problems. In the PPS algorithm particles are distributed in the exploration space consistently. In an atom how the electrons positioned in the centre accordingly particles are in the exploration space. Normally the movement of the particle is based on gradient and swarming motion $[16,17]$. When the gradient method failed then swarming is executed by inducing the particle shift towards the global most excellent position by modernizing the velocity. Validity of the Proposed Predestination of Particles Wavering Search (PPS) algorithm has been tested in standard IEEE $14,30,57,118,300$ bus systems and results show the projected PPS reduced the power loss effectively. 


\section{PROBLEM FORMULATION}

Objective of the problem is to reduce the true power loss:

$\mathbf{F}=\mathbf{P}_{\mathrm{L}}=\sum_{\mathrm{k} \in \mathrm{Nbr}} \mathbf{g}_{\mathrm{k}}\left(\mathbf{V}_{\mathrm{i}}^{2}+\mathbf{V}_{\mathrm{j}}^{2}-2 \mathbf{V}_{\mathrm{i}} \mathbf{V}_{\mathrm{j}} \cos \theta_{\mathrm{ij}}\right)$

Voltage deviation given as follows:

$F=P_{L}+\omega_{v} \times$ Voltage Deviation

Voltage deviation given by:

Voltage Deviation $\quad=\sum_{\mathbf{i}=1}^{N p q}\left|V_{i}-1\right|$

Constraint (Equality)

$\mathbf{P}_{\mathrm{G}}=\mathbf{P}_{\mathrm{D}}+\mathbf{P}_{\mathbf{L}}$

Constraints (Inequality)

$$
\begin{aligned}
& \mathbf{P}_{\text {gslack }}^{\text {min }} \leq \mathbf{P}_{\text {gslack }} \leq \mathbf{P}_{\text {gslack }}^{\text {max }} \\
& \mathbf{Q}_{\mathrm{gi}}^{\text {min }} \leq \mathbf{Q}_{\mathrm{gi}} \leq \mathbf{Q}_{\mathrm{gi}}^{\text {max }}, \mathbf{i} \in \mathbf{N}_{\mathrm{g}} \\
& \mathbf{V}_{\mathbf{i}}^{\text {min }} \leq \mathbf{V}_{\mathbf{i}} \leq \mathbf{V}_{\mathbf{i}}^{\text {max }}, \mathbf{i} \in \mathbf{N} \\
& \mathbf{T}_{\mathbf{i}}^{\text {min }} \leq \mathbf{T}_{\mathbf{i}} \leq \mathbf{T}_{\mathbf{i}}^{\text {max }}, \mathbf{i} \in \mathbf{N}_{\mathbf{T}} \\
& \mathrm{Q}_{\mathrm{c}}^{\min } \leq \mathrm{Q}_{\mathrm{c}} \leq \mathrm{Q}_{\mathrm{C}}^{\max }, \mathrm{i} \in \mathrm{N}_{\mathrm{C}}
\end{aligned}
$$

\section{PREDESTINATION OF PARTICLES WAVERING SEARCH ALGORITHM}

Predestination of Particles Wavering Search (PPS) algorithm has been modeled based on the motion of the particles in the exploration space. Particles will arbitrarily move in the exploration space in many algorithms which has been already applied to many optimization problems. In the PPS algorithm particles are distributed in the exploration space consistently. In an atom how the electrons positioned in the centre accordingly particles are in the exploration space. Normally the movement of the particle is based on gradient and swarming motion. Particles velocity has been initiated as follows,

$$
\text { velocity } y_{i}^{0}=\left[\frac{y_{\text {best }}-y_{i}^{0}}{2}\right]
$$

Particles are permitted to progress in steady velocity in gradient-based progress, but when the outcome is poor when compared to previous upshot, immediately particle rapidity will be upturned with semi of the magnitude and it will help to reach local optimal solution and it is expressed as wavering movement. Particle moves from point of slope $y_{1}$ to $y_{2}$ then it end's in negative fitness slope and when the particle velocity is multiplied by the value -0.50 , subsequently the particle moves from $y_{2}$ to $y_{3}$ then sequentially it end's in positive fitness slope, through this motion particle reach $y_{4}$ afterwards a negative fitness slope attained again by the particle then once again by -0.50 the particle velocity will be multiplied. Next at $y_{5}$ particle will attain, now the particle fitness will be positive slope, then in the same way particle continues its motion and it reach the point $y_{6}$. Once particle reaches the local optimal point $y_{\text {optimal }}$ then the velocity will be reversed again. When the gradient method failed then swarming is executed by inducing the particle shift towards the global most excellent position by modernizing the velocity as given below,

$$
\text { velocity } y_{i}^{t+1}=\text { velocity } y_{i}^{t}+\left[\frac{y_{\text {best }}-y_{i}^{t}}{2}\right]
$$


When the progress develop into constructive subsequently particle prolong to discover any more local optimal solution, and this procedure persist until maximum number of evaluation has been attained. Predestination of Particles Wavering Search (PPS) algorithm defined as follows,

Step 1 In the exploration space Initiate the particle's position with reference to boundary limits

Step 2: $\mathrm{i}=1 ; \mathrm{k}=1$

Step 3: Iterative procedure:

With respect to upper and lower boundaries particle positions are initiated

While ( $\mathrm{i}<=$ sum of particles)

Particles possible combinations has to be discovered

For $\mathrm{c}=1$ : sum of combinations

With respect to positions and combinations alter the positions of the particle $y_{i}$ as elevated values

$\mathrm{i}++$

End for

$\mathrm{k}++$

if ( $\mathrm{k}>$ dimensions) / when no boundary combinations are found then leave the loop /

Break

End if

End while

Step 4: Between two particles which has been already initiated some more particles are present, then factor based procedure is applied to reorganize the particle positions

Particles number are factorized

$\mathrm{f}=$ factor $(\mathrm{n}) ; \mathrm{n}=$ sum of particles $; \mathrm{f}$ is an array to store the factor values

Iterative procedure:

While $(\mathrm{i}<=\mathrm{n})$

For $c=1$ : sum of factors (with reference to length of " $\mathrm{f}$ ")

For $\mathrm{j}=1$ : dimensions $(\mathrm{p})$

For $\mathrm{i}=1: \mathrm{f}(\mathrm{c})$

$y_{i}(j)=\operatorname{minimum}(j)+k^{*}(\operatorname{maximum}(j)-\operatorname{minimum}(j)) /(f(c)+1)$

i++

End

End

if $\mathrm{i}>\mathrm{n}$ then when no boundary combinations are found then leave the loop

Repeat step 4 with Minimum and Maximum are exchanged

Break

End if

End for

End while

Then with suitable parameters projected Predestination of Particles Wavering Search (PPS) algorithm is applied to solve the optimal reactive power problem as shown below,

Step 1: Initialization of parameters

Step 2: In the exploration space Initiate the particle's position with reference to boundary limits

Step 3: Particles fitness values are computed and most excellent particle will be identified

Step 4: Velocity of the particles are initialized through velocity ${ }_{i}^{0}=\left[\frac{y_{\text {best }}-y_{i}^{0}}{2}\right]$

Step 5: Iterative procedure

While (computation number < maximum number of computation)

For $\mathrm{i}=1$; sum of particles

By augmenting the velocity to the present position determine new-fangled position

With reference to new-fangled position particle fitness should be calculated

Augmentations of computation counter, and then modernize global most excellent solution

When (slope $==$ unknown) then modernize slope of the particle with reference to new fitness to be positive or negative; Otherwise when (slope $==$ positive)

When (new-fangled fitness inferior than previous fitness); Then modernize velocity by " - velocity " ; modernize the slope with reference to new-fangled fitness to be negative; otherwise (slope $==$ negative)

When (new-fangled fitness inferior than the previous fitness)

Then modernize velocity by

velocity + (global most excellent position - present position/2)

Update slope to be unknown 
End if

End for

End while

Step 6: Global most excellent particle position found with fitness value

Step 7; Output the result

\section{SIMULATION RESULTS}

In standard IEEE 14 bus system the validity of the projected Predestination of Particles Wavering Search (PPS) algorithm has been tested, Table 1 shows the constraints of control variables Table 2 shows the limits of reactive power generators and comparison results are presented in Table 3.

Table 1. Constraints of control variables

\begin{tabular}{cccc}
\hline System & Variables & Minimum (PU) & Maximum (PU) \\
\hline \multirow{3}{*}{ IEEE 14 Bus } & Generator Voltage & 0.95 & 1.1 \\
& Transformer Tap & 0.9 & 1.1 \\
& VAR Source & 0 & 0.20 \\
\hline
\end{tabular}

Table 2. Constrains of reactive power generators

\begin{tabular}{cccc}
\hline System & Variables & Q Minimum (PU) & Q Maximum (PU) \\
\hline \multirow{3}{*}{ IEEE 14 Bus } & 1 & 0 & 10 \\
& 2 & -40 & 50 \\
& 3 & 0 & 40 \\
& 6 & -6 & 24 \\
& 8 & -6 & 24 \\
\hline
\end{tabular}

Table 3. Simulation results of IEEE-14 system

\begin{tabular}{ccccccc}
\hline Control variables & Base case & MPSO [18] & PSO [18] & EP [18] & SARGA [18] & PPS \\
\hline$V G-1$ & 1.060 & 1.100 & 1.100 & NR $^{*}$ & NR $^{*}$ & 1.012 \\
$V G-2$ & 1.045 & 1.085 & 1.086 & 1.029 & 1.060 & 1.013 \\
$V G-3$ & 1.010 & 1.055 & 1.056 & 1.016 & 1.036 & 1.019 \\
$V G-6$ & 1.070 & 1.069 & 1.067 & 1.097 & 1.099 & 1.024 \\
$V G-8$ & 1.090 & 1.074 & 1.060 & 1.053 & 1.078 & 1.003 \\
Tap 8 & 0.978 & 1.018 & 1.019 & 1.04 & 0.95 & 0.904 \\
Tap 9 & 0.969 & 0.975 & 0.988 & 0.94 & 0.95 & 0.903 \\
Tap 10 & 0.932 & 1.024 & 1.008 & 1.03 & 0.96 & 0.920 \\
$Q C-9$ & 0.19 & 14.64 & 0.185 & 0.18 & 0.06 & 0.145 \\
$P G$ & 272.39 & 271.32 & 271.32 & NR* & NR* & 271.60 \\
$Q G$ (Mvar) & 82.44 & 75.79 & 76.79 & NR* & NR* & 74.75 \\
Reduction in PLoss $(\%)$ & 0 & 9.2 & 9.1 & 1.5 & 2.5 & 24.67 \\
Total PLoss (Mw) & 13.550 & 12.293 & 12.315 & 13.346 & 13.216 & 10.206 \\
\hline NR & & & & & &
\end{tabular}

Then the projected Predestination of Particles Wavering Search (PPS) algorithm has been tested, in IEEE 30 Bus system. Table 4 shows the constraints of control variables, Table 5 shows the limits of reactive power generators and comparison results are presented in Table 6.

Table 4. Constraints of control variables

\begin{tabular}{cccc}
\hline System & Variables & Minimum (PU) & Maximum (PU) \\
\hline \multirow{3}{*}{ IEEE 30 Bus } & Generator Voltage & 0.95 & 1.1 \\
& Transformer Tap & 0.9 & 1.1 \\
& VAR Source & 0 & 0.20 \\
\hline
\end{tabular}

Table 5. Constrains of reactive power generators

\begin{tabular}{cccc}
\hline System & Variables & Q Minimum (PU) & Q Maximum (PU) \\
\hline \multirow{3}{*}{ IEEE 30 Bus } & 1 & 0 & 10 \\
& 2 & -40 & 50 \\
& 5 & -40 & 40 \\
& 8 & -10 & 40 \\
& 11 & -6 & 24 \\
& 13 & -6 & 24 \\
\hline
\end{tabular}


Table 6. Simulation results of IEEE -30 system

\begin{tabular}{ccccccc}
\hline Control variables & Base case & MPSO [18] & PSO [18] & EP [18] & SARGA [18] & PPS \\
\hline$V G-1$ & 1.060 & 1.101 & 1.100 & $\mathrm{NR}^{*}$ & $\mathrm{NR}^{*}$ & 1.013 \\
$V G-2$ & 1.045 & 1.086 & 1.072 & 1.097 & 1.094 & 1.014 \\
$V G-5$ & 1.010 & 1.047 & 1.038 & 1.049 & 1.053 & 1.061 \\
$V G-8$ & 1.010 & 1.057 & 1.048 & 1.033 & 1.059 & 1.005 \\
$V G-12$ & 1.082 & 1.048 & 1.058 & 1.092 & 1.099 & 1.024 \\
VG-13 & 1.071 & 1.068 & 1.080 & 1.091 & 1.099 & 1.043 \\
Tap11 & 0.978 & 0.983 & 0.987 & 1.01 & 0.99 & 0.904 \\
Tap12 & 0.969 & 1.023 & 1.015 & 1.03 & 1.03 & 0.912 \\
Tap15 & 0.932 & 1.020 & 1.020 & 1.07 & 0.98 & 0.906 \\
Tap36 & 0.968 & 0.988 & 1.012 & 0.99 & 0.96 & 0.905 \\
QC10 & 0.19 & 0.077 & 0.077 & 0.19 & 0.19 & 0.064 \\
QC24 & 0.043 & 0.119 & 0.128 & 0.04 & 0.04 & 0.103 \\
$P G$ (MW) & 300.9 & 299.54 & 299.54 & NR* & NR* & 298.62 \\
$Q G$ (Mvar) & 133.9 & 130.83 & 130.94 & NR* & NR* & 130.74 \\
Reduction in PLoss $(\%)$ & 0 & 8.4 & 7.4 & 6.6 & 8.3 & 18.41 \\
Total PLoss (Mw) & 17.55 & 16.07 & 16.25 & 16.38 & 16.09 & 14.319 \\
\hline NR
\end{tabular}

NR*-Not reported.

Then the proposed Predestination of Particles Wavering Search (PPS) algorithm has been tested, in IEEE 57 Bus system. Table 7 shows the constraints of control variables, Table 8 shows the limits of reactive power generators and comparison results are presented in Table 9.

Table 7. constraints of control variables

\begin{tabular}{cccc}
\hline System & Variables & Minimum (PU) & Maximum (PU) \\
\hline IEEE 57 Bus & Generator Voltage & 0.95 & 1.1 \\
& Transformer Tap & 0.9 & 1.1 \\
& VAR Source & 0 & 0.20 \\
\hline
\end{tabular}

Table 8. Constrains of reactive power generators

\begin{tabular}{cccc}
\hline System & Variables & Q Minimum (PU) & Q Maximum (PU) \\
\hline IEEE 57 Bus & 1 & -140 & 200 \\
& 2 & -17 & 50 \\
& 3 & -10 & 60 \\
& 6 & -8 & 25 \\
& 8 & -140 & 200 \\
& 9 & -3 & 9 \\
& 12 & -150 & 155 \\
\hline
\end{tabular}

Table 9. Simulation results of IEEE-57 system

\begin{tabular}{ccccccc}
\hline Control variables & Base case & MPSO [18] & PSO [18] & CGA [18] & AGA [18] & PPS \\
\hline$V G 1$ & 1.040 & 1.093 & 1.083 & 0.968 & 1.027 & 1.024 \\
$V G 2$ & 1.010 & 1.086 & 1.071 & 1.049 & 1.011 & 1.013 \\
$V G 3$ & 0.985 & 1.056 & 1.055 & 1.056 & 1.033 & 1.033 \\
$V G 6$ & 0.980 & 1.038 & 1.036 & 0.987 & 1.001 & 1.012 \\
$V G 8$ & 1.005 & 1.066 & 1.059 & 1.022 & 1.051 & 1.030 \\
$V G 9$ & 0.980 & 1.054 & 1.048 & 0.991 & 1.051 & 1.014 \\
$V G 12$ & 1.015 & 1.054 & 1.046 & 1.004 & 1.057 & 1.042 \\
Tap 19 & 0.970 & 0.975 & 0.987 & 0.920 & 1.030 & 0.953 \\
Tap 20 & 0.978 & 0.982 & 0.983 & 0.920 & 1.020 & 0.934 \\
Tap 31 & 1.043 & 0.975 & 0.981 & 0.970 & 1.060 & 0.920 \\
Tap 35 & 1.000 & 1.025 & 1.003 & NR* & NR* & 1.012 \\
Tap 36 & 1.000 & 1.002 & 0.985 & NR* & NR* & 1.004 \\
Tap 37 & 1.043 & 1.007 & 1.009 & 0.900 & 0.990 & 1.005 \\
Tap 41 & 0.967 & 0.994 & 1.007 & 0.910 & 1.100 & 0.990 \\
Tap 46 & 0.975 & 1.013 & 1.018 & 1.100 & 0.980 & 1.010 \\
Tap 54 & 0.955 & 0.988 & 0.986 & 0.940 & 1.010 & 0.973 \\
Tap 58 & 0.955 & 0.979 & 0.992 & 0.950 & 1.080 & 0.962 \\
Tap 59 & 0.900 & 0.983 & 0.990 & 1.030 & 0.940 & 0.961 \\
Tap 65 & 0.930 & 1.015 & 0.997 & 1.090 & 0.950 & 1.003 \\
Tap 66 & 0.895 & 0.975 & 0.984 & 0.900 & 1.050 & 0.952 \\
Tap 71 & 0.958 & 1.020 & 0.990 & 0.900 & 0.950 & 1.003 \\
Tap 73 & 0.958 & 1.001 & 0.988 & 1.000 & 1.010 & 1.004 \\
Tap 76 & 0.980 & 0.979 & 0.980 & 0.960 & 0.940 & 0.961 \\
\hline
\end{tabular}


Table 9. Simulation results of IEEE-57 system (Continued)

\begin{tabular}{ccccccc}
\hline Control variables & Base case & MPSO [18] & PSO [18] & CGA [18] & AGA [18] & PPS \\
\hline Tap 80 & 0.940 & 1.002 & 1.017 & 1.000 & 1.000 & 1.003 \\
$Q C 18$ & 0.1 & 0.179 & 0.131 & 0.084 & 0.016 & 0.172 \\
$Q C 25$ & 0.059 & 0.176 & 0.144 & 0.008 & 0.015 & 0.160 \\
$Q C 53$ & 0.063 & 0.141 & 0.162 & 0.053 & 0.038 & 0.142 \\
$P G$ (MW) & 1278.6 & 1274.4 & 1274.8 & 1276 & 1275 & 1270.12 \\
$Q G$ (Mvar) & 321.08 & 272.27 & 276.58 & 309.1 & 304.4 & 272.33 \\
Reduction in PLoss (\%) & 0 & 15.4 & 14.1 & 9.2 & 11.6 & 23.36 \\
Total PLoss (Mw) & 27.8 & 23.51 & 23.86 & 25.24 & 24.56 & 21.305 \\
\hline
\end{tabular}

NR*-Not reported.

Then the Predestination of Particles Wavering Search (PPS) algorithm has been tested, in IEEE 118 Bus system. Table 10 shows the constraints of control variables and comparison results are presented in Table 11.

Table 10. Constraints of control variables

\begin{tabular}{cccc}
\hline System & Variables & Minimum (PU) & Maximum (PU) \\
\hline IEEE 118 Bus & Generator Voltage & 0.95 & 1.1 \\
& Transformer Tap & 0.9 & 1.1 \\
& VAR Source & 0 & 0.20 \\
\hline
\end{tabular}

Table 11. Simulation results of IEEE-118 system

\begin{tabular}{|c|c|c|c|c|c|c|}
\hline Control variables & Base case & MPSO [18] & PSO [18] & PSO [18] & CLPSO [18] & PPS \\
\hline$V G 1$ & 0.955 & 1.021 & 1.019 & 1.085 & 1.033 & 1.013 \\
\hline$V G 4$ & 0.998 & 1.044 & 1.038 & 1.042 & 1.055 & 1.042 \\
\hline$V G 6$ & 0.990 & 1.044 & 1.044 & 1.080 & 0.975 & 1.024 \\
\hline$V G 8$ & 1.015 & 1.063 & 1.039 & 0.968 & 0.966 & 1.003 \\
\hline$V G 10$ & 1.050 & 1.084 & 1.040 & 1.075 & 0.981 & 1.012 \\
\hline$V G 12$ & 0.990 & 1.032 & 1.029 & 1.022 & 1.009 & 1.021 \\
\hline$V G 15$ & 0.970 & 1.024 & 1.020 & 1.078 & 0.978 & 1.034 \\
\hline$V G 18$ & 0.973 & 1.042 & 1.016 & 1.049 & 1.079 & 1.042 \\
\hline$V G 19$ & 0.962 & 1.031 & 1.015 & 1.077 & 1.080 & 1.034 \\
\hline$V G 24$ & 0.992 & 1.058 & 1.033 & 1.082 & 1.028 & 1.010 \\
\hline$V G 25$ & 1.050 & 1.064 & 1.059 & 0.956 & 1.030 & 1.031 \\
\hline$V G 26$ & 1.015 & 1.033 & 1.049 & 1.080 & 0.987 & 1.050 \\
\hline$V G 27$ & 0.968 & 1.020 & 1.021 & 1.087 & 1.015 & 0.902 \\
\hline$V G 31$ & 0.967 & 1.023 & 1.012 & 0.960 & 0.961 & 0.901 \\
\hline$V G 32$ & 0.963 & 1.023 & 1.018 & 1.100 & 0.985 & 0.913 \\
\hline$V G 34$ & 0.984 & 1.034 & 1.023 & 0.961 & 1.015 & 1.002 \\
\hline$V G 36$ & 0.980 & 1.035 & 1.014 & 1.036 & 1.084 & 1.001 \\
\hline$V G 40$ & 0.970 & 1.016 & 1.015 & 1.091 & 0.983 & 0.960 \\
\hline$V G 42$ & 0.985 & 1.019 & 1.015 & 0.970 & 1.051 & 1.001 \\
\hline$V G 46$ & 1.005 & 1.010 & 1.017 & 1.039 & 0.975 & 1.002 \\
\hline$V G 49$ & 1.025 & 1.045 & 1.030 & 1.083 & 0.983 & 1.003 \\
\hline$V G 54$ & 0.955 & 1.029 & 1.020 & 0.976 & 0.963 & 0.920 \\
\hline$V G 55$ & 0.952 & 1.031 & 1.017 & 1.010 & 0.971 & 0.961 \\
\hline$V G 56$ & 0.954 & 1.029 & 1.018 & 0.953 & 1.025 & 0.954 \\
\hline$V G 59$ & 0.985 & 1.052 & 1.042 & 0.967 & 1.000 & 0.963 \\
\hline$V G 61$ & 0.995 & 1.042 & 1.029 & 1.093 & 1.077 & 0.970 \\
\hline$V G 62$ & 0.998 & 1.029 & 1.029 & 1.097 & 1.048 & 0.982 \\
\hline$V G 65$ & 1.005 & 1.054 & 1.042 & 1.089 & 0.968 & 1.001 \\
\hline$V G 66$ & 1.050 & 1.056 & 1.054 & 1.086 & 0.964 & 1.002 \\
\hline$V G 69$ & 1.035 & 1.072 & 1.058 & 0.966 & 0.957 & 1.050 \\
\hline$V G 70$ & 0.984 & 1.040 & 1.031 & 1.078 & 0.976 & 1.034 \\
\hline$V G 72$ & 0.980 & 1.039 & 1.039 & 0.950 & 1.024 & 1.020 \\
\hline$V G 73$ & 0.991 & 1.028 & 1.015 & 0.972 & 0.965 & 1.013 \\
\hline$V G 74$ & 0.958 & 1.032 & 1.029 & 0.971 & 1.073 & 1.014 \\
\hline$V G 76$ & 0.943 & 1.005 & 1.021 & 0.960 & 1.030 & 1.005 \\
\hline$V G 77$ & 1.006 & 1.038 & 1.026 & 1.078 & 1.027 & 1.006 \\
\hline$V G 80$ & 1.040 & 1.049 & 1.038 & 1.078 & 0.985 & 1.003 \\
\hline$V G 85$ & 0.985 & 1.024 & 1.024 & 0.956 & 0.983 & 1.014 \\
\hline$V G 87$ & 1.015 & 1.019 & 1.022 & 0.964 & 1.088 & 1.013 \\
\hline$V G 89$ & 1.000 & 1.074 & 1.061 & 0.974 & 0.989 & 1.042 \\
\hline$V G 90$ & 1.005 & 1.045 & 1.032 & 1.024 & 0.990 & 1.031 \\
\hline$V G 91$ & 0.980 & 1.052 & 1.033 & 0.961 & 1.028 & 1.000 \\
\hline$V G 92$ & 0.990 & 1.058 & 1.038 & 0.956 & 0.976 & 1.031 \\
\hline
\end{tabular}

Real power loss diminution by predestination of particles wavering ... (Kanagasabai Lenin) 
Table 11. Simulation results of IEEE-118 system (Continued)

\begin{tabular}{|c|c|c|c|c|c|c|}
\hline Control variables & Base case & MPSO [18] & PSO [18] & PSO [18] & CLPSO [18] & PPS \\
\hline$V G 99$ & 1.010 & 1.023 & 1.037 & 0.954 & 1.088 & 1.003 \\
\hline$V G 100$ & 1.017 & 1.049 & 1.037 & 0.958 & 0.961 & 1.001 \\
\hline$V G 103$ & 1.010 & 1.045 & 1.031 & 1.016 & 0.961 & 1.010 \\
\hline$V G 104$ & 0.971 & 1.035 & 1.031 & 1.099 & 1.012 & 1.001 \\
\hline$V G 105$ & 0.965 & 1.043 & 1.029 & 0.969 & 1.068 & 1.050 \\
\hline$V G 107$ & 0.952 & 1.023 & 1.008 & 0.965 & 0.976 & 1.012 \\
\hline$V G 110$ & 0.973 & 1.032 & 1.028 & 1.087 & 1.041 & 1.014 \\
\hline$V G 111$ & 0.980 & 1.035 & 1.039 & 1.037 & 0.979 & 1.000 \\
\hline$V G 112$ & 0.975 & 1.018 & 1.019 & 1.092 & 0.976 & 1.091 \\
\hline$V G 113$ & 0.993 & 1.043 & 1.027 & 1.075 & 0.972 & 1.000 \\
\hline$V G 116$ & 1.005 & 1.011 & 1.031 & 0.959 & 1.033 & 1.001 \\
\hline Tap 8 & 0.985 & 0.999 & 0.994 & 1.011 & 1.004 & 0.943 \\
\hline Tap 32 & 0.960 & 1.017 & 1.013 & 1.090 & 1.060 & 1.000 \\
\hline Tap 36 & 0.960 & 0.994 & 0.997 & 1.003 & 1.000 & 0.951 \\
\hline Tap 51 & 0.935 & 0.998 & 1.000 & 1.000 & 1.000 & 0.933 \\
\hline Tap 93 & 0.960 & 1.000 & 0.997 & 1.008 & 0.992 & 1.002 \\
\hline Tap 95 & 0.985 & 0.995 & 1.020 & 1.032 & 1.007 & 0.970 \\
\hline Tap 102 & 0.935 & 1.024 & 1.004 & 0.944 & 1.061 & 1.001 \\
\hline Tap 107 & 0.935 & 0.989 & 1.008 & 0.906 & 0.930 & 0.942 \\
\hline Tap 127 & 0.935 & 1.010 & 1.009 & 0.967 & 0.957 & 1.000 \\
\hline QC 34 & 0.140 & 0.049 & 0.048 & 0.093 & 0.117 & 0.002 \\
\hline$Q C 44$ & 0.100 & 0.026 & 0.026 & 0.093 & 0.098 & 0.021 \\
\hline$Q C 45$ & 0.100 & 0.196 & 0.197 & 0.086 & 0.094 & 0.163 \\
\hline$Q C 46$ & 0.100 & 0.117 & 0.118 & 0.089 & 0.026 & 0.120 \\
\hline$Q C 48$ & 0.150 & 0.056 & 0.056 & 0.118 & 0.028 & 0.042 \\
\hline$Q C 74$ & 0.120 & 0.120 & 0.120 & 0.046 & 0.005 & 0.110 \\
\hline$Q C 79$ & 0.200 & 0.139 & 0.140 & 0.105 & 0.148 & 0.102 \\
\hline$Q C 82$ & 0.200 & 0.180 & 0.180 & 0.164 & 0.194 & 0.150 \\
\hline$Q C 83$ & 0.100 & 0.166 & 0.166 & 0.096 & 0.069 & 0.123 \\
\hline$Q C 105$ & 0.200 & 0.189 & 0.190 & 0.089 & 0.090 & 0.151 \\
\hline QC 107 & 0.060 & 0.128 & 0.129 & 0.050 & 0.049 & 0.133 \\
\hline$Q C 110$ & 0.060 & 0.014 & 0.014 & 0.055 & 0.022 & 0.001 \\
\hline PG(MW) & 4374.8 & 4359.3 & 4361.4 & $\mathrm{NR}^{*}$ & $\mathrm{NR}^{*}$ & 4362.10 \\
\hline QG(MVAR) & 795.6 & 604.3 & 653.5 & $\mathrm{NR}^{*}$ & $\mathrm{NR}^{*}$ & 610.11 \\
\hline Reduction in PLOSS (\%) & 0 & 11.7 & 10.1 & 0.6 & 1.3 & 13.84 \\
\hline Total PLOSS (Mw) & 132.8 & 117.19 & 119.34 & 131.99 & 130.96 & 114.418 \\
\hline
\end{tabular}

NR*-Not reported.

Then IEEE 300 bus system [18] is used as test system to authenticate the good performance of the Predestination of Particles Wavering Search (PPS) algorithm. Table 12 shows the comparison of real power loss obtained after optimization.

Table 12. Comparison of real power loss

\begin{tabular}{ccccc}
\hline Parameter & Method EGA [20] & Method EEA [20] & Method CSA [21] & PPS \\
\hline PLOSS (MW) & 646.2998 & 650.6027 & 635.8942 & 610.3371 \\
\hline
\end{tabular}

\section{CONCLUSION}

In this work Predestination of Particles Wavering Search (PPS) algorithm successfully solved the optimal reactive power problem. In the PPS algorithm particles are distributed in the exploration space consistently. In an atom how the electrons positioned in the centre accordingly particles are in the exploration space. Normally the movement of the particle is based on gradient and swarming motion. Particles are permitted to progress in steady velocity in gradient-based progress, but when the outcome is poor when compared to previous upshot, immediately particle rapidity will be upturned. In standard IEEE 14, 30, 57,118, 300 bus systems Predestination of Particles Wavering Search (PPS) algorithm have been tested and power loss has been reduced efficiently. 


\section{REFERENCES}

[1] K. Y. Lee, "Fuel-cost minimisation for both real and reactive-power dispatches," Proceedings Generation, Transmission and Distribution Conference, vol. 131, no. 3, pp. 85-93, 1984.

[2] Aoki, K., A. Nishikori and R.T. Yokoyama, "Constrained load flow using recursive quadratic programming, ”IEEE T. Power Syst., vol. 2, no. 1, pp. 8-16.1987.

[3] Kirschen, D.S. and H.P. Van Meeteren, "MW/voltage control in a linear programming based optimal power flow," IEEE T. Power Syst., vol. 3, no. 2, pp. 481-489.1988.

[4] Liu, W.H.E., A.D. Papalexopoulos and W.F. Tinney, "Discrete shunt controls in a Newton optimal power flow," IEEE T. Power Syst., vol. 7, no. 4, pp. 1509-1518, 1992.

[5] V. H. Quintana and M. Santos-Nieto, "Reactive-power dispatch by successive quadratic programming," IEEE Transactions on Energy Conversion, vol. 4, no. 3, pp. 425-435, 1989.

[6] V. de Sousa, E. Baptista, and G. da Costa, "Optimal reactive power flow via the modified barrier Lagrangian function approach,” Electric Power Systems Research, vol. 84, no. 1, pp. 159-164, 2012.

[7] Y. Li, X. Li, and Z. Li, "Reactive power optimization using hybrid CABC-DE algorithm," Electric Power Components and Systems, vol. 45, no. 9, pp. 980-989, 2017.

[8] Roy, Provas Kumar and Susanta Dutta, "Economic Load Dispatch: Optimal Power Flow and Optimal Reactive Power Dispatch Concept,” IGI Global, pp. 46-64, 2019.

[9] Christian Bingane, Miguel F., Anjos, Sébastien Le Digabel, "Tight-and-cheap conic relaxation for the optimal reactive power dispatch problem", IEEE Transactions on Power Systems, 2019,

[10] Dharmbir Prasad \& Vivekananda Mukherjee, "Solution of Optimal Reactive Power Dispatch by Symbiotic Organism Search Algorithm Incorporating FACTS Devices", IETE Journal of Research, vol. 64, no. 1, equatpp. 149-160, 2018.

[11] TM Aljohani, AF Ebrahim, O Mohammed Single, "Multiobjective Optimal Reactive Power Dispatch Based on Hybrid Artificial Physics-Particle Swarm Optimization,” Energies, vol. 12, no. 12, pp. 2333, 2019.

[12] Ram Kishan Mahate, \& Himmat Singh, "Multi-Objective Optimal Reactive Power Dispatch Using Differential Evolution," International Journal of Engineering Technologies and Management Research, vol. 6, no. 2, pp. 27-38, 2019.

[13] Yalçın, E, Taplamacıoğlu, M, Çam, E, "The Adaptive Chaotic Symbiotic Organisms Search Algorithm Proposal for Optimal Reactive Power Dispatch Problem in Power Systems,". Electrica, vol. 19, pp. 37-47, 2019.

[14] Mouassa, S. and Bouktir, T, "Multi-objective ant lion optimization algorithm to solve large-scale multi-objective optimal reactive power dispatch problem," COMPEL - The international journal for computation and mathematics in electrical and electronic engineering, vol. 38, no. 1, pp. 304-324, 2019.

[15] Tawfiq M. Aljohani, Ahmed F. Ebrahim \& Osama Mohammed, "Single and Multiobjective Optimal Reactive Power Dispatch Based on Hybrid Artificial Physics-Particle Swarm Optimization," Energies, MDPI, Open Access Journal, vol. 12, no. 12, pp. 1-24, 2019.

[16] Bartoccini, U., Carpi, A. Poggioni, V., Santucci, V., "Memes Evolution in a Memetic Variant of Particle Swarm Optimization," Mathematics, vol. 7, pp. 423, 2019.

[17] Fan, S. K. S. Jen, C. H., "An Enhanced Partial Search to Particle Swarm Optimization for Unconstrained Optimization," Mathematics, vol. 7, pp. 357, 2019.

[18] Ali Nasser Hussain, Ali Abdulabbas Abdullah and Omar Muhammed Neda, "Modified Particle Swarm Optimization for Solution of Reactive Power Dispatch," Research Journal of Applied Sciences, Engineering and Technology, vol. 15, no. 8, pp. 316-327, 2018.

[19] IEEE, “The IEEE-test systems", www.ee.washington.edu/trsearch/pstca/.1993.

[20] S.S. Reddy, et al., "Faster evolutionary algorithm based optimal power flow using incremental variables," Electrical Power and Energy Systems, vol. 54, pp. 198-210, 2014

[21] S. Surender Reddy, "Optimal Reactive Power Scheduling Using Cuckoo Search Algorithm," International Journal of Electrical and Computer Engineering (IJECE), vol. 7, no. 5, pp. 2349-2356. 2017. 